\title{
UN MODELO DE EVALUACIÓN DE LA CALIDAD DE LAS BIBLIOTECAS UNIVERSITARIAS DIGITALES BASADO EN TÉCNICAS DIFUSAS
}

\author{
A QUALITY EVALUATION MODEL FOR DIGITAL UNIVERSITY LIBRARIES BASED ON \\ FUZZY TOOLS
}

E. Herrera-Viedma, S. Alonso - viedma, salonso@decsai.ugr.es Dpto. de Ciencias de la C. e I.A.- Fac. Biblioteconomía y Documentación

Universidad de Granada - España

Javier López Gijón, B. Ávila - jgijon@ugr.es

Facultad de Biblioteconomía y Documentación

Universidad de Granada - España

Josefina Vílchez Pardo - jvpardo@ugr.es

Facultad de Biblioteconomía y Documentación

Universidad de Granada - España

Comente este artigo no blog Ebibli = http://encontros-bibli-blog.blogspot.com/

\section{Resumen}

Se presenta un modelo para la evaluación de la calidad de las bibliotecas universitarias digitales basado en técnicas difusas. El modelo contiene tanto criterios objetivos como subjetivos. Usa información difusa lingüística para la representación de las valores de calidad y operadores de agregación de información lingüística para obtenerlos.

Palabras clave: Evaluación de calidad. Bibliotecas universitarias. Bibliotecas digitales. Indicadores de calidad. Modelado lingüístico difuso.

\section{INTRODUCCIÓN}

La evaluación de los sistemas de información es una actividad necesaria que forma parte de su ciclo de vida y que contribuye a mejorarlos. Las bibliotecas universitarias son los centros de información donde se almacena y distribuye la información en el contexto universitario. La evaluación de las bibliotecas es necesaria por diversas razones: evaluamos para conocer los puntos fuertes y débiles de la biblioteca, para conocer el nivel de rendimiento y saber cómo seria posible mejorarlo, también evaluamos para saber el grado de cumplimiento de los objetivos que consigue el centro y por tanto poder conocer el grado de eficacia que tiene, es decir, poner en relación los resultados obtenidos con los objetivos que se habían marcado. Pero si todas estas razones para evaluar son importantes hemos de reconocer que la razón fundamental, como nos dice Marchionini (2000) y la mayoría de los autores consultados, es que "el objetivo de una evaluación es conocer el impacto que la biblioteca tiene en el medio social". El único problema de conocer este impacto de la biblioteca, que otros autores llaman beneficio, sobre su medio es que no es fácil de medir. 
Las Nuevas Tecnologías de la Información y Comunicación (NTIC) están revolucionando las pautas de convivencia en la sociedad actual, afectando tanto a instituciones y empresas públicas y/o privadas, como a los ciudadanos en general. En particular, la introducción de las NTIC ha contribuido sustancialmente a la modernización de las bibliotecas universitarias. Se comenzó con la automatización de los catálogos de las bibliotecas universitarias, y poco a poco, con su creciente aplicación (el desarrollo de las redes abiertas de ordenadores, el éxito de Internet, el crecimiento de WWW y sus capacidades hipertextuales) está apareciendo un nuevo concepto de biblioteca universitaria, "La Biblioteca Universitaria Digital" (Cook et al., 2003; García, 2001). En esta nueva concepción digital de biblioteca universitaria, los libros y revistas impresos serán sustituidos por libros y revistas digitales, los tradicionales depósitos, reemplazados por los repositorios electrónicos de información, y el acceso a la lectura se hará desde cualquier lugar (bien en las propias salas de lectura o bien en otros espacios como por ejemplo la propia casa personal), principalmente desde los ordenadores personales u otros dispositivos, a través de las redes abiertas de información (García, 2001).

En estos y los próximos años tendremos unas Bibliotecas Universitarias Híbridas en las que convivan los dos modelos de biblioteca, el tradicional y el digital, pero con una clara tendencia a desarrollar la componente digital de la biblioteca. Podemos decir que nada de lo que había en la biblioteca tradicional se ha perdido. Pero ahora hay más, nos encontramos ante un sistema superior. Está tendencia está dando lugar a la aparición de nuevos problemas que habrá que resolver (García, 2001): de soportes físicos y de líneas de telecomunicaciones; de diseño informáticos para gestionar sistemas distribuidos e interfaces inteligentes de acceso a la información; de definición de normas informáticas y de edición electrónica; de estándares bibliotecarios; de disposiciones legales que regulen la propiedad intelectual de la información digital; y de nuevos modelos o normas de evaluación de la calidad de las bibliotecas que recojan su nueva dimensión digital, dado que no podemos olvidar, como dice el Prof. Saracevic (2000, p.350-369), que "una biblioteca digital es mucho más que una colección de textos digitalizados y otros objetos", y por tanto se acabará generando una nueva realidad no comparable con la concepción tradicional de biblioteca.

Ante esta situación, como una primera aproximación a la evaluación de la calidad de las bibliotecas universitarias digitales, en esta contribución presentamos un modelo para la Evaluación de la Calidad de la Bibliotecas Universitarias Digitales. Las principales características de este modelo son las siguientes: 
1. Presenta un esquema de evaluación que incorpora tanto criterios objetivos como subjetivos, los cuales pueden ser ponderados dependiendo de su importancia en el esquema global de evaluación.

2. Está basada en Técnicas Difusas de Computación con Palabras, pues los valores de calidad asociados a cada biblioteca son etiquetas lingüísticas obtenidas mediante la agregación desarrollada por los operadores lingüísticos LOWA (Linguistic Ordered Weigthed Averaging) (Herrera et al., 1996, p.175-190) y LWA (Linguistic Weighted Averaging) (Herrera y Herrera-Viedma, 1997, p.646-656).

\section{LA EVALUACIÓN DE LA CALIDAD DE LAS BIBLIOTECAS UNIVERSITARIAS DIGITALES}

La evaluación de las bibliotecas universitarias digitales es actualmente una práctica poco desarrollada en España, aunque también lo es en la mayor parte de los países que cuentan con estas nuevas bibliotecas. Y aunque como experiencias concretas encontramos muy pocas, sí abundan más los autores que plantean cómo deben hacerse estas evaluaciones, los aspectos que hay que tener en cuenta, o los criterios que se deben seguir. Hay dos problemas que hacen que la evaluación de bibliotecas universitarias digitales en España sea una práctica poco extendida: la escasez de datos sobre el desarrollo digital de las bibliotecas universitarias y el poco acuerdo que hay sobre los indicadores que se deben calcular.

En el año 2000, para dar un norma general de evaluación del sistema bibliotecario español, el Grupo de Evaluación de REBIUN publicó la Lista de Indicadores de Rendimiento para la Evaluación de Bibliotecas Universitarias, que fue distribuida a todas las bibliotecas universitarias, no parece que el resultado haya sido satisfactorio, pues las bibliotecas siguen manifestando, si vemos sus autoevaluaciones, que se necesita más concreción y acuerdo en los indicadores que se deben calcular.

Por otro lado, el problema de los pocos estudios que hay sobre calidad de las bibliotecas universitarias digitales no se da solamente en España, sino también a nivel internacional, pues como Chao nos comenta (2002, p.169-194) "bastantes estudios se han realizado sobre criterios de evaluación de fuentes de información y sitios web, pero son pocos los estudios que han dado normas o criterios para medir la calidad de los sitios web de las bibliotecas". Algunos de los estudios que podemos resaltar son los siguientes: 
John Carlo Bertot (2004) consideró que las herramientas actuales para la evaluación de la calidad en las bibliotecas digitales en un entorno de red son insuficientes, pues no se adecuan correctamente a la evaluación de las mismas. Según él, los modelos de evaluación deben basarse en herramientas que indagan más específicamente en vez de preguntar de manera general. Por tanto, propone ir más allá de las medidas del tipo de resultados para entender la calidad, el valor y el impacto percibidos del servicio y los recursos de la biblioteca digital; seguir desarrollando medidas, métodos e indicadores para los servicios y recursos de la biblioteca digital (diferentes de la biblioteca tradicional); y considerar medidas y enfoques que incluyen al usuario. La propuesta de Bertot nos parece de lo más acertado y representa un buen punto de partida para plantearse cómo deberíamos evaluar bibliotecas digitales.

Otro modelo para la evaluación de un sistema de biblioteca digital es el que expone Fred Heath (2003) y sus colaboradores. Describen, comparan y analizan dos proyectos de evaluación de bibliotecas digitales: el protocolo LibQUAL+(Health et al., 2003) y el proyecto CAPM (Comprehensive Access to Printed Materials) (Cook et al., 2003); y proponen un modelo mixto de esos dos proyectos. LibQUAL+ proporciona herramientas para evaluar la calidad de los servicios (e-servicios) de las bibliotecas digitales que identifican las carencias en la difusión de sus servicios. A partir de este análisis, la metodología CAPM facilita el desarrollo de los servicios de las bibliotecas digitales basándose en las preferencias y expectativas de los usuarios. Usa modelos multi-atributos y de afirmación de preferencias. El modelo de evaluación híbrido que los autores desarrollan en base a esos proyectos consiste en aplicar LibQUAL+ como identificador de las carencias de los servicios de la biblioteca digital. Esta identificación se convierte en la base para el uso del modelo CAPM, mediante el cual se les pide a los usuarios que prioricen las soluciones propuestas que les parecen más adecuadas para contribuir a la eliminación de las lagunas en la calidad de los servicios de la biblioteca digital. En definitiva, es un modelo de calidad totalmente subjetivo que evalúa la tolerancia del usuario en cuanto a lo que está dispuesto a aceptar en la calidad de los servicios, midiendo lo deseado y lo percibido por el individuo. ECABUD también permite una evaluación subjetiva, a partir de un cuestionario que contestan los usuarios.

Young (1998, p.66-69) hace hincapié en los problemas que plantea la evaluación de material electrónico, en su artículo insiste en la necesidad de un análisis descriptivo de las estadísticas de tecnologías de la información electrónica, esencial para mejorar la eficacia de la organización, la eficiencia y la productividad. Piensa que es fundamental " $e l$ establecimiento de una metodología estadística normativa y la creación de definiciones, 
estructuras y categorías que describan estos recursos, que son esenciales para la gestión y la programación de bibliotecas". Opina que la rapidez con la que se está desarrollando la biblioteca digital pone en cuestión las estadísticas que se utilizan en las bibliotecas tradicionales y la propia noción de evaluación. Cree Young (1998, p.66-69) que se va a necesitar "la elaboración nuevas formas de contar, basadas más en el ejemplo del comercio electrónico que en las normas de estadísticas de bibliotecas". Lo que el autor nos dice es que no está tan claro que para las bibliotecas digitales sirvan los mismos datos, indicadores y modelos que se usan para la biblioteca tradicional, en lo que estamos totalmente de acuerdo con Young. En su artículo plantea una serie de mediciones que deberían hacerse para conocer y evaluar la biblioteca digital, entre las que podemos destacar: medidas basadas en las transacciones, medidas basadas en la duración de las conexiones, medidas basadas en el cálculo de costos, y medidas basadas en la utilización. ECABUD ha intentado integrar en su modelo de evaluación la mayor parte de los indicadores que desarrolla Young (1998, p.6669).

En "Assessing the quality of academics libraries on the web: the development and testing of criteria" (Chao, 2002, p.169-194) presentó un instrumento para evaluar la calidad de las bibliotecas universitarias en la Web. Chao, al igual que casi todos los autores que hemos visto, mantiene que "hay una laguna de guías o criterios de autoridad para ayudar a los profesionales de bibliotecas a definir como la calidad de los sitios web de las bibliotecas universitarias pueden ser medidos y mejorados para ofrecer un mejor servicio". Su modelo de evaluación consta de 16 indicadores de calidad que tras una minuciosa experimentación fueron seleccionados desde una de una colección de de 68 indicadores de calidad tomados de los distintos modelos existentes en la literatura que fueron valorados por un grupo amplio de expertos bibliotecarios. Estos 16 criterios que él propone para evaluar la calidad son: contenido, presentación, información institucional, servicios, títulos y cabeceras, credibilidad, diseño gráfico, capacidades de búsqueda, navegabilidad, autoría, inclusión de colecciones especiales, facilidad de uso y ayuda al uso, compatibilidad, información sobre los enlaces, integración, y velocidad.

Otro autor que también ha tratado el tema de la evaluación en profundidad es Marchionini (2000, p.304-333) que nos dice que una evaluación debe "especificar cuales son los procesos de investigación (métricos y de procedimiento), cual es su misión y sus características más sobresalientes, y el contexto(s) en el cual opera". Opina que "un modelo de evaluación de centros de información debe incluir mediciones de cuatro clases: costos de 
entrada, salidas (cuantitativas, cualitativas, disponibilidad, accesibilidad), eficacia (percepción de los servicios por los usuarios, satisfacción de usuarios) y competencia o propiedad (personal, tipos de necesidades de información, comportamiento de usuarios)".

Saracevic (2000, p.350-369) realizó un estudio sobre el estado de la cuestión en la investigación sobre bibliotecas digitales y propuso su propio modelo conceptual para la evaluación de las bibliotecas digitales. Apunta que los estudios de usuarios son criterios importantes e imprescindibles para la evaluación de las bibliotecas digitales, pero no son suficientes para su evaluación global. Insiste en la necesidad de un modelo amplio. Reconoce como requerimientos básicos para la evaluación de bibliotecas digitales los criterios siguientes: Lo que se quiere evaluar, el contexto de la evaluación (objetivos), los criterios que reflejan el rendimiento relativo a cada uno de los objetivos, las medidas que se van a emplear para registrar el rendimiento, y la metodología de la evaluación.

Como podemos observar la investigación en materia de evaluación de la calidad de las bibliotecas digitales está enfocada principalmente en los modelos de usuarios, que van evolucionando. Sin embargo, la mayoría de los autores coinciden en que éste no puede ser el único criterio para la evaluación de una biblioteca digital, la cual tiene que ser analizada de forma global y conjunta. También observamos que casi la totalidad opinan que los modelos de evaluación no están claros todavía y que se necesitan más estudios empíricos y evaluaciones concretas para ir desarrollando un modelo válido y aceptable por todos.

Basándonos en los trabajos que acabamos de comentar, hemos diseñado nuestro modelo para evaluar la calidad de las bibliotecas universitarias digitales, que presentamos en la siguiente sección.

\section{UN MODELO DE EVALUACIÓN DE LA CALIDAD DE LAS BIBLIOTECAS UNIVERSITARIAS DIGITALES}

La incorporación de las NTCI en las bibliotecas universitarias está dando lugar a que aparezcan nuevos aspectos de las mismas que ya no pueden ser evaluados mediante los esquemas tradicionales de evaluación de las bibliotecas, los cuales están basados fundamentalmente en criterios cuantitativos.

Con la progresiva aplicación de las NTCI en la actividad de las bibliotecas universitarias se está produciendo un incremento considerable de la interacción biblioteca-usuarios, tanto en cantidad como en calidad de servicios y flujo de información. Este hecho no pasa 
desapercibido en los equipos de gestión de las bibliotecas y cada vez está más consolidada la idea de que un buen modelo de evaluación de la calidad de las bibliotecas universitarias digitales no puede dejar a un lado la opinión de los usuarios, como se pone de manifiesto en los diferentes modelos de evaluación de calidad de las bibliotecas que hemos analizado en la sección anterior.

Sin embargo, aunque hay nuevos aspectos imposibles de evaluar con los indicadores tradicionales que requieren la creación de nuevos indicadores de calidad, y una tendencia a incorporar en los modelos de evaluación de calidad criterios cualitativos relacionados con los usuarios, esto no significa que haya que renunciar a todos los criterios cuantitativos tradicionales.

Por todo ello, proponemos que la evaluación de calidad de las bibliotecas universitarias digitales debe basarse tanto en criterios objetivos como en cualitativos subjetivos, de entre los cuales tenemos algunos nuevos (por ejemplo, el indicador Megas per cápita) y otros que son extensiones digitales de criterios objetivos tradicionales (consultas per cápita a la biblioteca universitaria digital). Además la mayoría de criterios seleccionados, están orientados a los usuarios, los objetivos reflejando lo que los usuarios hacen (por ejemplo, consultas per cápita a la biblioteca universitaria digital) y los subjetivos reflejando lo que opinan o dicen (por ejemplo, el criterio de valor añadido).

Asumiendo lo expuesto, definimos nuestro modelo de evaluación de bibliotecas universitarias digitales que presenta tanto criterios objetivos como subjetivos, que pueden ser ponderados, que está orientado a usuario y que usa técnicas difusas lingüísticas para obtener los valores de calidad. Este modelo de evaluación de calidad contiene dos elementos:

1. Un esquema de evaluación: Reúne los indicadores de calidad usados en la evaluación de las bibliotecas universitarias digitales, tanto los objetivos como los subjetivos.

2. El método de computación de los valores de calidad: Es la técnica de computación usada para a partir de las distintas valoraciones asignadas a los indicadores de calidad obtener los valores de calidad globales asociados a las bibliotecas universitarias.

Ambos son analizados en las siguientes subsecciones.

\subsection{Esquema de evaluación}

Sobre los objetivos que el conjunto de indicadores o criterios de evaluación debería cumplir:

"el sistema de indicadores que se adopte en un Servicio de Bibliotecas universitarias debe ofrecer, de manera rápida y sin grandes esfuerzos, datos 
globales, para el conjunto del Servicio y datos individuales, por biblioteca y análisis comparativos y de evolución entre unidades o bibliotecas". (Alonso et al., 1999)

Justamente esto es lo que pretendemos en nuestro modelo, pues diseñamos un esquema de evaluación que permite conocer la situación de una biblioteca determinada, y permite comparar la situación de varias.

Sobre la evaluación de los servicios de la biblioteca hay distintas teorías que se pueden seguir con vistas a poder extraer modelos y principios generales. Según algunos autores una de las más apropiadas puede ser la Teoría General de Sistemas. Una de las características generales de la Teoría de sistemas es que los elementos individuales pueden ser combinados para producir algo que es más que la suma de las partes. O lo que es lo mismo el conocido punto de la Teoría de Sistemas que nos dice que el todo es mayor que la suma de las partes. De acuerdo a ella, para un conocimiento sistémico de la biblioteca se necesitan múltiples medidas de todo el sistema bibliotecario, y el punto de vista contrario, por el que si usamos gran variedad de mediciones para evaluar conseguimos un conocimiento más global de la biblioteca.

Por ello, en primer lugar hemos intentado que nuestro esquema de evaluación sea muy amplio y abarque la mayor parte de los componentes de una biblioteca universitaria digital. Igualmente hemos intentado combinar diferentes medidas, aceptando que estas combinaciones o niveles en la evaluación nos pueden aportar información extra y que no se recoge en el nivel inferior.

Por tanto, el esquema de evaluación que proponemos está compuesto tanto de criterios cuantitativos objetivos como de cualitativos subjetivos, con especial atención a aquellos que bien sirven para observar la conducta de los usuarios o bien para recoger sus opiniones o juicios de valor. El esquema de evaluación presenta una estructura jerárquica compuesta de dos niveles:

\section{Primer Nivel:}

Contiene los criterios cuantitativos objetivos obtenidos midiendo directamente características del sistema. Para establecer los criterios cuantitativos seguimos a autores como Ingwersen (1998), Chao (2002) o Thelwall (2002), que intentan generar una base matemática que dé validez a las evaluaciones que plantean. Nos fijamos en la actividad de una biblioteca analizada como un sistema de información usando la Teoría de Sistemas: a partir de unas entradas del sistema, que normalmente se miden como inversiones económicas en las distintas 
facetas de la biblioteca, se realizan unos procesos bibliotecarios que generan unos recursos y activos en la entidad bibliotecaria con los que se originan unas salidas del sistema relacionadas con los servicios que presta a la comunidad universitaria, es decir, con la interacción biblioteca-usuario. Adicionalmente, también se pueden incluir entre las salidas del sistema aquellos beneficios que se generan a la sociedad no-universitaria. Para trabajos de bibliotecas universitarias digitales las entradas, los procesos de entidad y las salidas del sistema podrían medirse, dependiendo del modelo seguido, como:

a) ENTRADAS: Euros por habitante (habitantes $=$ alumnos + profesores); \% de gasto en recursos-e sobre \% total en gasto en recursos informativos; gasto en recursos-e per capita (pc); gasto en equipamiento informático pc (o por 100).

b) PROCESOS DE ENTIDAD: Megas pc (un indicador parecido seria el equivalente a volúmenes por habitante); revistas-e/investigador; revistas-e/habitante; puestos de lecturae por habitante (o por 100); terminales para uso del catálogo (exclusivamente) pc (o por 100 ); ordenadores (sin conexión a internet) pc (o por 100); digitalización del fondo antiguo.

c) SALIDAS: Circulación por habitante medida por número de documentos-e bajados; costo por unidad que circula; tasa de rotación (total circulación/total colección); artículos-e bajados/investigador; documentos-e bajados/habitante; búsquedas en revistase/investigador; visitas a la web pc; consultas catálogo pc; consultas-búsquedas a la biblioteca digital (BD) /investigador; consultas-búsquedas BD /habitante; porcentaje de accesos externos a la BD.

Nuestro esquema de evaluación presenta 8 criterios. De entre los indicadores objetivos de actividad de las entradas, de los procesos de entidad y de la salida hemos seleccionado los siguientes 4: accesos pc a la BD, consultas pc, puestos de acceso pc, MB pc. Para medir el estado de digitalización de la biblioteca universitaria hemos incorporado el indicador porcentaje de revistas digitales. Para medir la visibilidad externa de la biblioteca universitaria hemos definido el indicador porcentaje de accesos externos. Además, de estos indicadores de calidad objetivos también introducimos dos que tienen que ver con las posibilidades de visibilidad externa de la biblioteca universitaria digital: posibilidad de multilenguaje y factor de impacto del Web site de la biblioteca. 


\section{Segundo Nivel:}

Contiene criterios subjetivos obtenidos directamente de las opiniones proporcionadas por los usuarios sobre el funcionamiento del sistema. Algunos autores (Huang et al., 1999; Lee et al., 2002) presentan un entorno de evaluación de calidad para el diseño de sistemas de información bajo la idea de que la calidad de los sistemas de información no puede ser evaluada dejando a un lado la opinión de los consumidores de información, que son los usuarios. Este entorno contempla cuatro categorías de evaluación de calidad en donde se pueden clasificar los distintos criterios de evaluación: calidad intrínseca, calidad contextual, calidad de la representación y calidad de accesibilidad. Usando este entorno de calidad de información vamos a definir el conjunto de criterios subjetivos que componen nuestro esquema de evaluación de calidad para las bibliotecas universitarias digitales. Dado que nosotros queremos diseñar un modelo de evaluación de calidad orientado al usuario, vamos a definir criterios subjetivos que sean fácilmente comprensibles por los usuarios y en un número no muy alto para que no provoque el rechazo de los usuarios. Por tanto, nuestro esquema de evaluación en su vertiente cualitativa establece cuatro categorías de calidad para caracterizar la calidad de las bibliotecas universitarias digitales entre las cuales se reparten los criterios de evaluación de calidad que consideramos:

a) CALIDAD INTRÍNSECA: Medimos la calidad intrínsica o precisión de las bibliotecas universitarias digitales en términos de si los usuarios encuentran o no lo que buscan. Por tanto, en esta categoría definimos el siguiente criterio cualitativo: Encuentra lo que busca.

b) CALIDAD CONTEXTUAL: Para evaluar los aspectos informativos de las bibliotecas universitarias digitales desde una perspectiva de la tarea consideramos los siguientes criterios cualitativos: grado de satisfacción global con la biblioteca, presenta utilidades de valor añadido, cobertura de la biblioteca universitaria sobre la materia que se busca, $y$ servicio de información sobre nuevas adquisiciones.

c) CALIDAD DE REPRESENTACIÓN: La calidad de representación de las bibliotecas universitarias digitales es evaluada de acuerdo a los siguientes criterios cualitativos: La comprensibilidad de la biblioteca digital y la formación recibida para entender la biblioteca.

d) CALIDAD DE ACCESIBILIDAD: La calidad de accesibilidad de las bibliotecas universitarias digitales es medida atendiendo a los siguientes criterios cualitativos: Variedad de herramientas de búsqueda de información, Navegabilidad de la biblioteca digital, infraestructura informática de la biblioteca digital, $y$ tiempo de respuesta del sistema bibliotecario digital ante una consulta.

\subsection{Método de computación de los valores de calidad}

En esta subsección vamos a definir el método que usamos para computar los valores de calidad globales que caracterizan la calidad de las bibliotecas universitarias digitales a partir 
de las evaluaciones obtenidas para cada uno de los criterios de calidad que componen el esquema de evaluación definido en la anterior.

Primeramente, hemos de comentar como obtenemos los valores para los diferentes criterios. Los valores para los criterios cuantitativos son obtenidos desde la observación directa de la actividad de las bibliotecas universitarias digitales. Por otro lado, para obtener los valores para los criterios cualitativos tenemos que recurrir a definir una encuesta que los usuarios deben de rellenar después de haber usado la biblioteca universitaria digital. Para representar las opiniones dadas por los usuarios en la encuesta usamos información lingüística difusa representada mediante un enfoque lingüístico difuso ordinal (Herrera et al., 1996; Herrera y Herrera-Viedma, 1997; Herrera-Viedma et al., 2006).

En un enfoque lingüístico difuso ordinal asumimos un conjunto de etiquetas finito y totalmente ordenado en el sentido normal y con un cardinal impar (7 o 9 etiquetas).

La etiqueta central $S=\left\{s_{i}, i \in H=0, \mathrm{~K}, T\right\}$ representa una valoración de “aproximadamente 0.5 " y el resto se sitúan simétricamente alrededor de ella. La semántica de las etiquetas se define en base a la estructura ordenada que forman el

conjunto de etiquetas considerando que el par $\left(\mathrm{s}_{\mathrm{i}}, \mathrm{s}_{T-\mathrm{i}}\right)$ es igualmente informativo. Cada etiqueta tiene asociado un número difuso sobre $[0,1]$, que se define mediante una función de pertenencia trapezoidal representada por una 4-tupla $\left(\mathrm{a}_{\mathrm{i}}, \mathrm{b}_{\mathrm{i}}, \alpha_{\mathrm{i}} \beta_{\mathrm{i}}\right)$ (los primeros dos parámetros

$$
\begin{aligned}
& \text { 1.-Orden }: s_{i} \geq s_{j} \quad \text { si } \quad i \geq j . \\
& \text { 2.-Negación : } \operatorname{Neg}\left(s_{i}\right)=s_{j} \text {, con } j=T-i \text {. } \\
& \text { 3.-Máximo : } \operatorname{MAX}\left(s_{i}, s_{j}\right)=s_{i} \text { si } s_{i} \geq s_{j} \text {. } \\
& \text { 4.-Mínimo }: \operatorname{MIN}\left(s_{i}, s_{j}\right)=s_{i} \text { si } s_{j} \leq s_{j} \text {. }
\end{aligned}
$$

indican el intervalo en el que el valor de pertenencia es 1; el tercero y cuarto indican la amplitud a la izquierda y a la derecha de la distribución). Además, requerimos las siguientes propiedades:

Por ejemplo, podemos usar el siguiente conjunto de nueve etiquetas con su semántica asociada para evaluar las variables lingüísticas: $\{\mathrm{T}=$ Total $=(1,1,0,0)$, $\mathrm{EA}=$ Extremadamente_Alto $=(.98, .99, .05, .01), \mathrm{MA}=$ Muy_Alto $=(.78, .92, .06, .05), \mathrm{A}=$ Alto $=$ $(.63, .80, .05, .06), \mathrm{M}=$ Medio $=(.41, .58, .09, .07), \mathrm{B}=$ Bajo $=(.22, .36, .05, .06), \mathrm{MB}=$ Muy_Bajo $=(.1, .18, .06, .05), \mathrm{EB}=$ Extremadamente_Bajo $=(.01, .02, .01, .05), \mathrm{N}=$ Nulo $=$ $(0,0,0,0)\}$. 
Para combinar y agregar información lingüística se hace necesario la definición de adecuados operadores de agregación. En nuestro modelo usaremos el operador LOWA (Herrera et al., 1996) para combinar información lingüística con igual importancia y el operador LWA (Herrera y Herrera-Viedma, 1997) para combinar información lingüística con diferente importancia.

Los valores de calidad global que obtenemos son de naturaleza lingüística. El proceso de cálculo de los valores de calidad presenta tres pasos:

Primer Paso: Cálculo de un valor de calidad global objetivo. Consiste en la agregación de los valores de los criterios objetivos a través del operador LWA. Para llevar a cabo esta computación usaremos una función de conversión de valores numéricos en valores lingüísticos llamada Label, que asigna etiqueta de $\mathrm{S}$ a un valor numérico $\mathrm{r} \in[0,1], \quad \operatorname{Label}(\mathrm{r})=$ $\operatorname{Sup}_{\mathrm{q}}\left\{\left\{\mathrm{s}_{\mathrm{q}} \in \mathrm{S}: \mu \mathrm{s}_{\mathrm{q}}(\mathrm{r})=\operatorname{Sup}_{\mathrm{v}} \mu \mathrm{s}_{\mathrm{v}}(\mathrm{r})\right\}\right\}$.

Segundo Paso: Cálculo de un valor de calidad global subjetivo. Para cada usuario se calcula un valor de calidad parcial subjetivo con respecto a las opiniones que expresó en su encuesta. Para ello usamos el LWA. Luego se computa el valor de calidad global subjetivo agregando los valores de calidad parciales subjetivos obtenidos para cada usuario por medio del operador LOWA.

Tercer Paso: Cálculo de un valor de calidad global. El valor global de calidad de una biblioteca universitaria digital lo obtenemos agregando los valores de calidad globales objetivos y subjetivos usando el operador LOWA.

\section{CONCLUSIONES}

En este trabajo hemos presentado el modelo de evaluación de calidad de las bibliotecas universitarias digitales. Este modelo está implementado en la herramienta software ECABUD (http://sci2s.ugr.es/evaluacionBibliotecas/), una herramienta diseñada para evaluar la calidad de las bibliotecas universitarias digitales españolas. Esta herramienta constituye un primer paso hacia un sistema global de análisis y gestión de las bibliotecas digitales del futuro.

ECABUD nos permite medir la calidad de las bibliotecas digitales independientemente y al mismo tiempo nos permite compararlas. Es una herramienta orientada a usuario, tanto en la concepción de los criterios que usa para evaluar la calidad como en las técnicas que usa para representar la información, que la hace más amigable. 


\section{REFERENCIAS}

ALONSO, J.; ECHEVARRÍA, M.J. y MARTÍN, S. La gestión de las bibliotecas universitarias: Indicadores para su evaluación. En: Seminario de Indicadores en la Universidad: Información y Decisiones, León, 1999.

BERTOT, J.C. Assessing Digital Library Services: Approaches, Issues, and Considerations. En: Proceedings of the International Symposium on Digital Libraries and Knowledge Communities in Networked Information Society DLKC'04, March 2 - 5, 2004, Tsukuba, Ibaraki (Japan), 2004.

CHAO, H. Assessing the quality of academic libraries on the Web: The development and testing of criteria. Library \& Information Science Research, v. 24, 2002, p. 169-194.

COOK, C. et al. Developping a National Science Digital Library (NSDL) LibQUAL+ Protocol: An E-service for Assessing the Library of the $21^{\text {st }}$ Century. En: NSDL Evaluation Workshop, 2003.

GARCÍA, E. y GARCÍA, L.A. La Biblioteca Digital. Madrid: Arco Libros, 2001.

HEATH, F. et. al. Emerging Tools for Evaluating Digital Library Services: Conceptual Adaptations of LibQUAL+ and CAPM. Journal of Digital Information, v. 4, n. 2, Article No. 170, 2003. Disponible en: <www.libqual.org $>$

HERRERA, F.; HERRERA-VIEDMA, E. y VERDEGAY, J.L. Direct Approach Processes in Group Decision Making using Linguistic OWA operators. Fuzzy Sets and Systems, v. 79, 1996, p. 175-190.

HERRERA, F. y HERRERA-VIEDMA, E. Aggregation Operators for Linguistic Weighted Information. IEEE Transactions on Systems, Man and Cybernetics, v. 27, 1997, p. 646656.

HERRERA-VIEDMA, E. et al. Evaluating the Information Quality of Web Sites: A Methodology Based on Fuzzy Computing with Words. Journal of American Society for Information Science and Technology, v. 57, n. 4, 2006, p. 538-549.

HUANG, K.; LEE, Y.W. y WANG, R.Y. Quality information and knowledge. Upper Saddle River, NJ: Prentice Hall, 1999.

INGWERSEN, P. The calculation of Web Impacts Factors. Journal of Documentation, v. 54 , n. 2,1998 , p. 236-243.

LEE, Y. W. et al.. AIMQ: A methodology for information quality assessment. Information \& Management, v. 40, n. 2, 2002, p.133-146.

MARCHIONINI, G. Evaluating digital libraries: A longitudinal and multifaceted view. Library Trends, v. 49, n. 2, 2000, p. 304-333. 
SARACEVIC, T. Digital library evaluation: Toward an evolution of concepts. Library Trends, v. 49, n. 3, 2000, p. 350-369.

THELWALL, M. A comparison of sources of links for academic Web impact factor calculations. Journal of Documentation, v. 58, n. 1, 2002, p. 66-78.

YOUNG, P. L'evaluation des services électroniques en bibliotèque: les statistisques. Bulletin des Bibliotheques de France, v. 43, n. 3, 1998, p. 66-69.

\begin{abstract}
A quality evaluation model for digital university libraries based on fuzzy tools is presented. This model offers both objective and subjective quality criteria. It uses a fuzzy linguistic modelling to represent quality values and linguistic aggregation operators to obtain them.
\end{abstract}

KEY-WORDS: Quality evaluation. University libraries. Digital libraries. Quality criteria. Fuzzy linguistic modelling.

Originais recebidos em 26 de maio de 2006. 